\title{
Prosodic Transfer in the Beginning-and Advanced-Level Chinese Learners' Production of English Word Stress
}

\author{
Xing-rong $\mathrm{GUO}^{1, \mathrm{a}}$,Xiao-xiang $\mathrm{CHEN}^{1, \mathrm{~b}^{*}}$ and Yi-ming $\mathrm{GUO}^{2, \mathrm{c}}$ \\ ${ }^{1}$ College of Foreign Languages, Hunan University, Changsha, China \\ ${ }^{2}$ School of Economics and Management, Shanghai Maritime University, \\ Shanghai, China \\ aguoxingrong356@163.com, bhuda319@163.com, ymguo@shmtu.edu.cn
}

Keywords: Prosodic transfer, English word stress, Production, Proficiency, Chinese learners.

\begin{abstract}
English second language (L2) learners often experience difficulties in producing native-like English lexical stress. It is unknown which acoustic correlates (F0, duration and intensity) are the most problematic for Chinese learners of different proficiency levels. The present study investigates the prosodic transfer effects of first language (L1) on the production of English lexical stress by 10 beginning-level and 10 advanced-level Chinese learners of English. Results showed that L2 learners produced less native-like stress patterns in terms of acoustic correlates, values varied according to their L2 proficiency. Although all Chinese learners of English used F0, duration, and intensity to identify stress, they performed differently on the use of duration, possibly due to L1 tonal transfer.
\end{abstract}

\section{Introduction}

A foreign accent is always observed from some adult L2 learners due to L1 influence. In suprasegmental study, lexical stress has been a research focus. Chinese is a tone language with four regular tones and a neutral tone. Unlike Mandarin Chinese, English is a typical stress language with dynamic interaction of fundamental frequency (F0), duration and intensity [1].

When considering L2 English stress production, both L1 interference and L2 effect are reported by previous research $[1,2]$.Ueyama raised the issue of L1 prosodic transfer and reported that L1 prosodic features can be transferred into L2 speech development, especially the timing feature [2]. Difference in timing types can result in the durational discrepancy between stressed and unstressed syllables. It has been proved that the timing difference may cause the problems in L2 speech production [3].

The problems of Chinese learners faced in learning English stress were assumed to arise from tonal transfer [3].Nguyen found that due to L1 tonal transfer, the be inners and the advanced Vietnamese learners could use F0 and intensity to signal stress in a native way, though the advanced speakers could produce native-like duration stress contrast, beginners failed to differentiates stress by duration [1]. Zhang et al. reported that Mandarin speakers could produce native-like intensity and duration, but they produced stressed syllables with a higher F0 than native speakers [3].

Much work has been done investigating the production of lexical stress in English, However, little research focus on the acoustic correlates of stress in Chinese ESL learners, especially in the perspective of different L1 proficiency levels. This study tries to explore L1transfer effect on the acoustic realization of English lexical stress by Chinese EFL speakers with different proficiency levels. 


\section{Methods}

\section{Participants}

The participants were divided into 3 groups: 10 Native American English speakers, 10 beginning-level Chinese learners of English and 10 advanced-level Chinese learners of English. There were 5 males and 5 females in each group. The Chinese learners' English proficiency were judged by the Language Experience and Proficiency Questionnaire (LEAP-Q) [4] and the Lexical Test for Advanced Learners of English (LexTALE) [5]. Then the Chinese learners were divided into two groups: the beginning-level and the advanced-level groups. None of the participants reported being diagnosed with any language or reading disorders.

\section{Stimuli}

5 real minimal stress pairs were used in the experiment: "contract, object, subject, and permit, suspect". Two disyllabic English words with either a trochaic or iambic stress pattern are used as stimuli since they are the most common types of English words (CELEX database).

\section{Procedure}

In the training session, participants were instructed the stress rule and provided enough time for practice. In the experimental session, they read stimuli 3 times. Only the third repetition was analyzed. The following acoustic parameters were measured of individual vowels by using Praat (version 5.1.37): F0, duration and intensity.

\section{Analysis and Results}

\section{Average Fo}

Results of the average F0 values are shown in Figure 1.

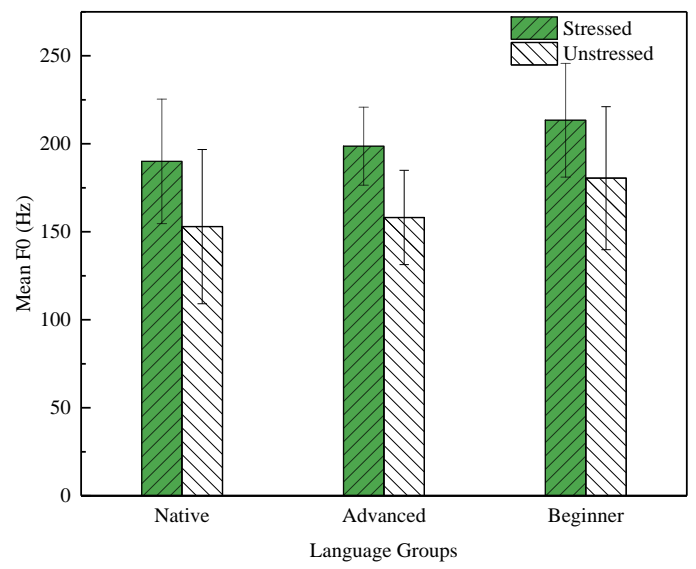

Figure 1. Average F0 Values of the three groups

As shown in Figure 1, the F0 results of Factorial ANOVA showed significant main effects for language groups $[F(1,27)=7.566, p=0.001]$, gender $[F(1,27)=266.405$, $p<0.001]$ and stress patterns $[F(1,27)=39.616, p<0.001]$. Besides, significant interactions were found between gender and stress patterns $[F(1,27)=5.379$, $p=0.023]$, but no other significant interactions $(p>0.05)$. The Posthoc (Turkey HSD) tests demonstrated F0 of the stressed syllable for three groups was significantly higher compared than the unstressed ones. There was a significant effect of gender: F0 values of female (mean $226 \mathrm{~Hz}$ ) were significantly higher than that of male speakers $(136 \mathrm{~Hz})$. There is no significant difference among the native English speakers and the advanced speakers $(p=0.348)$, but significant difference were found between the advanced-level 
speakers and the beginning-level learners $(p<0.05)$. In the stressed syllables, the beginning speakers produced significantly higher F0 than English speakers $(p<0.001)$. Thus, the language-group difference of F0 was: beginning-level group $>$ advanced-level group> native English group.

\section{Average Duration}

Results of the average duration values are shown in Figure 2.

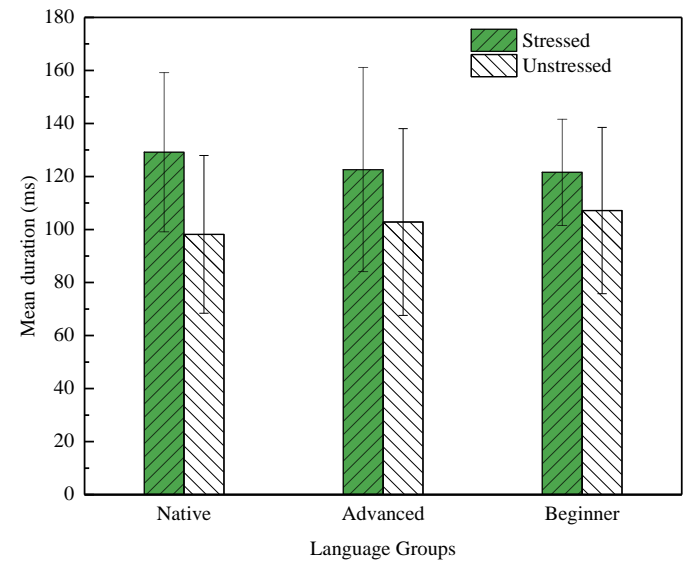

Figure 2. Average duration values of the three groups

As shown in Figure 2, result revealed a significant main effect of language groups $[F(1,27)=7.538, p=0.001]$, gender $[F(1,27)=9.487, p=0.003]$ and stress patterns $[F$ $(1,27)=12.344, p<0.001]$, but no other significant interactions $(p>0.05)$. Post hoc tests demonstrated duration of the stressed syllable for all language groups was significantly longer than the unstressed syllables. There is significant difference between the native speakers and the advanced speakers $(p=0.001)$, the native speakers and the beginning-level learners $(p=0.022)$, but no significant difference were found between the advanced and the beginning-level learners $(p<0.725)$. Thus, the duration difference was in the order: native group > beginning-level group $>$ advanced-level group.

\section{Average Intensity}

Results of the average intensity values are shown in Figure 3.

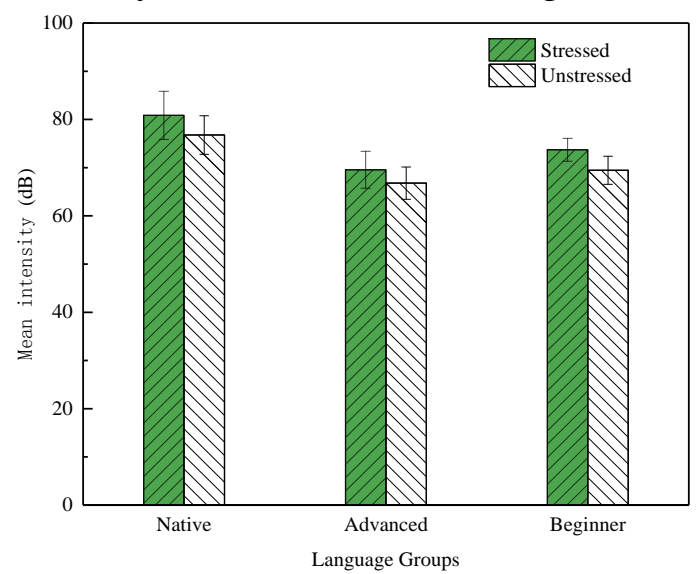

Figure 3. Average intensity values of the three groups

As shown in Figure 3, the results of intensity values showed significant main effects for language groups $[F(1,27)=50.289, p<0.001]$, gender $[F(1,27)=13.018$, $p<0.001]$ and stress patterns $[F(1,27)=15.921, p<0.001]$. There were significant difference between groups and gender $(p<0.001)$, but no other significant interactions $(p>0.05)$. Post hoc tests demonstrated intensity of the stressed syllable for the 3 
groups was significantly stronger than the unstressed ones. There is significant difference between the native speakers and the advanced speakers $(p<0.001)$, the native speakers and the beginners $(p<0.001)$, the advanced-level learners and the beginning-level learners $(p<0.001)$. Thus, the group difference was: native group $>$ beginning-level group $>$ advanced-level group.

\section{Discussion}

The experimental results showed that, similar to the native American English speakers, the beginning-level and the advanced-level ESL learners were all able to produce stressed syllables with higher F0 than unstressed syllables. However, the amount of F0 in the stressed syllables of ESL learners exceeded more than native American English speakers. This result is consistent with the reported by [3]. Such F0 deviation may be attributable to two explanations: 1) the anthropomorphic discrepancy between Chinese and American speakers lead the F0 difference. Asian speakers had an overall smaller laryngeal framework than Caucasian speakers [6]; 2) Chinese is a tonal language. The lexical tones in Chinese may influence the beginning-level and the advanced-level learners in producing English lexical stress, particularly in using F0 for stressed syllables [7].

Results also showed that both the beginning-level and the advanced-level speakers could differentiate stress by duration cue. Stressed syllable were significantly longer than unstressed ones. Such lengthening of the stressed syllables by the beginning-level and the advanced-level learners is similar to that of native speakers. This finding is consistent with [3]. Whereas, compared with native speakers, both the beginning-level and the advanced-level learners produced the stressed and the unstressed syllables with relatively shorter duration. Such difference may be related to Chinese' syllable-timed feature. In Chinese, there is a relatively regular interval between two syllables [7, 8]. It may be very difficult for ESL learners of a syllable-timed language to produce English stress pattern by manipulating duration in a native way.

The difference in intensity between the stressed and unstressed syllables suggested that the beginning-level and the advanced-level learners could use intensity to differentiate stress. However, they were significant different from native speakers. This finding was different from Zhang et al. [3], who found that Mandarin speakers produced intensity in a native fashion. Intensity serves as the secondary cue for Mandarin lexical stress [9]. Chinese speakers' use lower intensity in English lexical stress probably resulted from the less prominent role it plays in Mandarin. Results from experimental data show that there is prosodic transfer in the speech of L2 learners.

The results generally support the advanced-level learners could differentiate stress by F0, but the beginning-level learners used too much F0 to signal stress. Such finding suggests that the active role of F0 as the primary cue in Chinese tones facilitated their production of L2 stress contrasts. Due to the L1 influence, both the beginning-level and the advanced-level groups have some problems in producing English word stress. Though the advanced-level learners could use F0 to produce stress patterns like native speakers, but they failed to differentiate English stress in terms of duration and intensity. The beginning-level learners failed to explore all the three cues. Such finding suggests a negative L1 transfer effect on L2 stress production, since duration does not play an active role in Chinese tonal distinctions, so both the 
beginning-level and the advanced-level learners failed to encode this cue in produce native-like stress contrasts.

\section{Conclusion}

In sum, native speakers and nonnative speakers employ prosodic correlates in a different fashion that are optimally tuned to L2 learners' L1 phonological system. Although the beginning-level and the advanced-level learners failed to produce native-like stress contrasts in terms of these cues, it does not represent that they cannot encode these cues. The present study suggest L2 teachers give explicitly teaching to L2 learners about these features, so as to help them grasp the L2 prosodic characteristics faster. In particular, they should pay more attention to control L1 effect on L2.

\section{Acknowledgments}

This study was funded by National Social Science Foundation (14BYY144) and China Postdoctoral Science Foundation (2016M590348).

\section{References}

[1] A.T. Nguyễn, C.L.J. Ingram, J.R. Pensalfini, Prosodic transfer in Vietnamese acquisition of English contrastive stress patterns. Journal of Phonetics. 36 (2008) 158-190.

[2] M. Ueyama, Prosodic transfer: An acoustic study of L2 English vs. L2 Japanese. Aiche Org, 2000.

[3] Y. Zhang, S.L. Nissen, A.L. Francis, Acoustic characteristics of English lexical stress produced by native Mandarin speakers. J. Acoust. Soc. Am. 123 (2008) 4498-4513.

[4] V. Marian, Blumenfeld HK, Kaushanskaya M. The Language Experience and Proficiency Questionnaire (LEAP-Journal of Speech, Language, and Hearing Research Q): Assessing language profiles in bilinguals and multilinguals. 50 (2007) 940-967.

[5] K. Lemhöfer, M. Broersma, Introducing LexTALE: A quick and valid lexical test for advanced learners of English. Behavior Research Methods. 44 (2012) 325-343.

[6] B. Yang, A comparative study of American English and Korean vowels produced by male and female speakers. Journal of Phonetics. 24 (1996) 245-261.

[7] M.L. Ng, Y.Chen, Proficiency in English sentence stress production by Cantonese speakers who speak English as a second language (ESL). International journal of speech-language pathology. 13 (2011) 526-535.

[8] P. Roach, English phonetics and phonology: a practical course: Cambridge University Press.

[9] D.H. Whalen, Y. Xu, Information for Mandarin tones in the amplitude contour and in brief segments. Phonetica. 49 (1992) 25-47. 